https://doi.org/10.15407/ujpe66.9.739

S.L. PARNOVSKY

Astronomical Observatory of Taras Shevchenko Kyiv National University

(3, Observatorna Str., Kyiv 04058, Ukraine; e-mail: par@observ.univ.kiev.ua)

\title{
POSSIBLE MODIFICATION OF THE STANDARD COSMOLOGICAL MODEL TO RESOLVE A TENSION WITH HUBBLE CONSTANT VALUES
}

\begin{abstract}
The tensions concerning the values of Hubble constant obtained from the early and the late Universe data pose a significant challenge to modern cosmology. Possible modifications of the flat homogeneous isotropic cosmological $\Lambda C D M$ model are considered, in which the Universe contains the dark energy, cold baryonic matter, and dark matter. They are based on general relativity and satisfy two requirements: (1) the value of the Hubble constant calculated from the value of the Hubble parameter at the recombination by formulas of the flat $\Lambda$ CDM model, should be equal to 92\% of the one based on low-redshift observations; (2) deviations from the $\Lambda C D M$ model should not lead to effects that contradict astronomical observations and estimations obtained thereof. The analysis showed that there are few opportunities for the choice. Either we should consider DM with negative pressure $-\rho_{d m} c^{2} \ll p_{d m}<0$, which weakly affects the evolution of the Universe and the observed manifestations of DM, or we should admit the mechanism of generation of new matter, for example, by the dark energy decay.

Ke ywords: cosmology, Hubble constant, tension, dark matter, dark energy.
\end{abstract}

\section{Introduction}

A great interest among cosmologists was caused by tensions between the values of the Hubble constant obtained from the observations of the early and the late Universe indicated recently in [1]. I simply note that the estimation $H_{0}=67.4 \mathrm{~km} \mathrm{~s}^{-1} \mathrm{Mpc}^{-1}$ obtained from observations in the recombination era accounts for about $92 \%$ of the average of the estimations based on observations of not very distant objects, $H_{0}=73.3 \mathrm{~km} \mathrm{~s}^{-1} \mathrm{Mpc}^{-1}$. The corresponding difference is at the level of $4 \sigma-6 \sigma$, which, according to [1], should be classified as something from a discrepancy or a problem to a crisis. They are robust to the exclusion of any single method, team or source.

It is known that the expansion rate of the Universe is characterized by the time-dependent Hubble

(C) S.L. PARNOVSKY, 2021

ISSN 2071-0194. Ukr. J. Phys. 2021. Vol. 66, No. 9 parameter $H$. Its current value is called the Hubble constant and is denoted by $H_{0}$. The value of the Hubble constant is calculated from the Hubble parameter measured in some era. This requires knowledge of both the characteristics of this era, usually its redshift $z$, and the cosmological model to relate these values.

Estimations of the Hubble constant obtained by different methods are given in [1]. Most measurements of the Hubble parameter occur at distances, which are small by cosmological standards. They have small redshifts, and these measurements relate to the late Universe. However, a few measurements relate to the early Universe, more precisely to the recombination era (redshift $z=z_{r} \approx 1100$ ).

First of all, these are CMB data from the Planck satellite [2] and data from the Dark Energy Survey Year 1 clustering combined with data on the weak lensing, baryon acoustic oscillations, and Big Bang nucleosynthesis [3]. 
Naturally, the differences could be explained by measurement errors, including errors in observational data, their processing, or interpretation, or by an influence of some poorly accounted factors. That would mean that cosmology is not yet an exact science, as it pretends to be. For the purpose of this work, I choose to ignore possible issues with observational data and consider them to be correct. This article demonstrates that the contradiction can be possibly eliminated by a modification of the standard $\Lambda \mathrm{CDM}$ cosmological model. It is clear that deviations from the $\Lambda \mathrm{CDM}$ model can not be large, because it is compatible with most observations.

I considered an isotropic homogeneous cosmological model, in which the Universe is filled with the dark energy (DE), dark matter (DM), and cold baryonic matter. The Universe has passed the era of recombination, so the influence of radiation and ultrarelativistic particles can be neglected. However, this model must be different from the $\Lambda \mathrm{CDM}$ model, so that it can explain the $8 \%$ difference in the Hubble constants at $z=z_{r}$ and $z=0$.

I consider three possible modifications of it. In the first one, the dark energy with an arbitrary equation of state is used instead of the cosmological constant $\Lambda$. In the second, the dark matter is not pressureless, although its pressure is small in comparison with the energy density. An example of such matter is the so-called warm dark matter (WDM). A hypothetic WDM was introduced earlier in astrophysics to solve some problems associated with the clustering on subgalactic scales and the formation of halos [4]. WDM is often mentioned in explanations of a monochromatic signal around $3.5 \mathrm{keV}$ in the spectrum of $\mathrm{X}$-ray emissions from galaxy clusters like Perseus and Centaurus observed by the XMM-Newton $[5,6]$. The current most popular candidates for WDM particles are sterile neutrinos [7], gravitinos, non-thermally produced WIMPs, and other particles beyond the Standard Model.

The third modification is associated with the possibility of the transition or decay of DE into matter or vice versa. The question is being investigated as to whether any of these modifications can explain the Hubble constant tension without contradicting other astronomical observations. The following step will be to consider options that meet this criterion in more detail.

\section{The Choice of a Cosmological Model Can Affect the Values of the Hubble Constant}

I consider the flat relativistic isotropic homogeneous cosmological model with a scale factor $a(z)$ at the interval of redshifts $z=a_{0} / a-1$ from the recombination era $\left(z=z_{r}\right)$ to the present epoch $(z=0)$. I assume that the Universe consists of dark energy (DE), cold baryonic matter, and dark matter (DM). The influence of radiation and ultrarelativistic particles can be neglected. The energy-momentum tensor of each component has a diagonal form $\mathbf{T}=\operatorname{diag}\left(\rho c^{2}, p, p, p\right)$ in the comoving frame. Note that I define the pressure of DM and DE just as a component of the corresponding energy-momentum tensor.

The Hubble parameter change law is described by the first Friedmann equation [8]

$$
\begin{aligned}
& H^{2}=\frac{8 \pi G}{3} \rho=\frac{8 \pi G}{3}\left(\rho_{m}+\rho_{d e}\right)= \\
& =H_{0}^{2}\left(\frac{\rho_{m}}{\rho_{m 0}} \Omega_{m 0}+\frac{\rho_{d e}}{\rho_{d e 0}} \Omega_{d e 0}\right) .
\end{aligned}
$$

Here, $\rho$ and $\Omega$ are the density and the density parameter, the subscripts $m$ and de denote matter and dark energy, the subscript 0 denotes the current value of the corresponding quantity, and $G$ is the gravitational constant. I assume that the Universe contains only matter, both dark and baryonic ones and DE. This formula can also be obtained in the framework of nonrelativistic cosmology [9].

The Hubble constant value is calculated from the Hubble parameter obtained by processing the observational data by Eq. (1) for the $\Lambda$ CDM model. It assumes that the dark energy is a pure cosmological constant quatity with constant density, and both baryonic and cold dark matters are pressureless. For this model, I use the standard subscript $\Lambda$ instead of de. Using the dependences of $\rho_{\Lambda}=$ const and $\rho_{m}=$ $=\rho_{m 0}(1+z)^{3}$, one gets

$H^{2}=H_{0}^{2}\left(\Omega_{\Lambda 0}+(1+z)^{3} \Omega_{m 0}\right)$.

According to the Planck satellite observations, the parameters of cosmological constant and matter density in the modern era are $\Omega_{\Lambda 0}=0.68 \pm 0.02$ and $\Omega_{m 0}=0.32 \pm 0.02[2]$, and their sum is fixed to 1 in the flat model. Note that these quantities are of the same order.

ISSN 2071-0194. Ukr. J. Phys. 2021. Vol. 66, No. 9 
If the real cosmological model differs from the $\Lambda \mathrm{CDM}$ one, we used (2) instead of (1). In this case, we would get not the value $H_{0}$, but rather the product $A(z) H_{0}$ :

$H=H_{0} \sqrt{\frac{\rho_{m}}{\rho_{m 0}} \Omega_{m 0}+\frac{\rho_{d e}}{\rho_{d e 0}} \Omega_{d e 0}}=$

$=A(z) H_{0} \sqrt{\Omega_{\Lambda 0}+(1+z)^{3} \Omega_{m 0}}$

with the factor

$A(z)=\sqrt{\frac{\frac{\rho_{m}}{\rho_{m 0}} \Omega_{m 0}+\frac{\rho_{d e}}{\rho_{d e 0}} \Omega_{d e 0}}{\Omega_{\Lambda 0}+(1+z)^{3} \Omega_{m 0}}}$.

If the $\Lambda \mathrm{CDM}$ model is correct, we have $A(z)=1$. If it is not correct, then $A(z)$ is almost equal to 1 for the late Universe, but could differ from 1 for the early one. Let us try to explain the discrepancies in the values of the Hubble constant using Eq. (4). To explain the results of the article [1], we need to provide $A\left(z_{r}\right) \approx 0.92$. It is clear that this means to go beyond the $\Lambda$ CDM-model. We try to look for such modification which could provide the condition for $A\left(z_{r}\right)$ (the condition $A(0)=1$ is done automatically). An additional requirement is small deviations from the model, especially during the period of existence and evolution of galaxies and stars, i.e. from the reionization era or so-called "cosmic dawn", $z<z_{g} \approx 11$.

\section{DE Cannot Solve the Problem}

Let us start with the letter $\Lambda$ in the name of the model and consider the dark energy with variable density instead of the cosmological constant. The CDM part remains the same. We get $A\left(z_{r}\right)<1$, if $\rho_{d e}\left(z_{r}\right)<$ $<\rho_{d e 0}$, but we cannot obtain $A\left(z_{r}\right)=0.92$. Indeed,

$A(z)>\sqrt{\frac{(1+z)^{3} \Omega_{m 0}}{\Omega_{\Lambda 0}+(1+z)^{3} \Omega_{m 0}}}$,

but this value is less than 1 by no more than $10^{-8}$ at $z \approx 1100$. This is caused by the value of the $(1+z)^{3}$ factor at large $z$. It's a dead end.

\section{DM with Non-Zero Pressure}

Now, I abandon the letters CDM in the model title and leave only the letters DM. This is not about the effects of electromagnetic radiation or ultrarelativistic particles and neutrinos. They exist, but their share is so small that, even at $z \sim 1100$, they do not provide the desired value of $A(z)$. So, let us temporalily forget about them and suppose that matter is composed of ordinary matter and dark matter. We know enough about the baryonic matter to be sure that it can be considered as pressureless one. Its density $\rho_{b}=\rho_{b 0}(1+z)^{3}$.

But, we actually know just a bit about the DM. Suppose that it has some pressure $p_{d m}=w(z) \rho_{d m} c^{2}$ which can affect the evolution of its density $\rho=$ $=\rho_{0} F(z)(1+z)^{3}$. For matter and DE (see $\S 2.7 .4$ in $[9])$, the first law of thermodynamics gives

$\frac{d \rho}{\rho+p c^{-2}}=-\frac{d V}{V}=-3 \frac{d a}{a}$.

Here, $V \propto a^{3}$ is a volume of a part of the space expanding with the Universe, $a=a_{0} /(1+z)$ is the scale factor. This equation and the corresponding EoS specify the dependences of the densities of all components on $z$. I assume that each of the components, i.e. DE, baryonic and dark matter, expands adiabatically. This means, in particular, that none of them could decay or transform into another one. It is not difficult to find the relation

$F(z)=\exp \left(3 \int_{0}^{z} \frac{w(\xi) d \xi}{\xi+1}\right)$.

If the density of matter $\rho_{m}$ is equal to the sum of the densities of baryonic $\rho_{b}$ and dark matter $\rho_{d m}$, then, from (4), we obtain the expression for $A(z)$ at large $z$, when it is possible to neglect the terms with subscripts de and $\Lambda$ :

$A(z)=\sqrt{\frac{F(z) \Omega_{d m 0}+\Omega_{b 0}}{\Omega_{m 0}}}$.

I used the value $\Omega_{b 0}=0.16 \Omega_{m 0}$ based on the Planck data [2] to get the rough estimation $F\left(z_{r}\right)=0.82$ from the condition $A\left(z_{r}\right)=0.92$. From (7), it is seen that this is impossible for $w(z) \geq 0$. That is, the DM must have a negative pressure for at least some time interval after the recombination. So, it cannot be called WDM.

\subsection{The case of the simplest EoS}

First, I consider the DM with the simplest and most popular equation of state (EoS),

$p_{d m}=w \rho_{d m} c^{2}$ 
where the subscript dm means dark matter, $\rho_{d m}$ and $p_{d m}$ are the DM density and pressure, $c$ is the speed of light, and $w=$ const. I calculate the parameter $w$ which could explain the difference in the estimated values of the Hubble constant obtained from highand low-redshift data. From (9) and (6), we obtain the expression $F(z)=(1+z)^{3 w}$. One gets the rough estimation $w \approx-0.009$ from $F\left(z_{r}\right)=0.82$.

It is clear that neither classical particles, nor bosons, nor fermions can have a negative pressure. So, DM should have, in this case, a completely different nature than ordinary matter. On the other hand, the introduction of such a small negative pressure has little effect on the numerous astronomical manifestations of DM. I mean the rotation curves of galaxies, estimations of virial masses of galaxy clusters, gravitational lensing, galaxy cluster mergers like the Bullet Cluster (1E 0657-56), and so on (see Chap. 4 in [9]). It could affect the results of modeling of the large-scale structure formation, however.

This pressure with $|w| \ll 1$ also weakly affects the evolution of the Universe. I will show that the changes are insignificant taking the age of the Universe as an example. It can be found from (1) for the flat model with cosmological constant, cold baryonic matter, and DM with EoS (9). This age is equal to

$T=H_{0}^{-1} \int_{0}^{1} \frac{u^{0.5+1.5 w} d u}{\sqrt{u^{3(1+w)} \Omega_{\Lambda 0}+u^{3 w} \Omega_{b 0}+\Omega_{d m 0}}}$.

It is easy to calculate that, at $w=-0.009$, it is 13.9 billion years or the $100.76 \%$ of the age of the Universe for the $\Lambda \mathrm{CDM}$ model, which is equal to 13.8 billion years. As one can see, the difference from the case of the $\Lambda \mathrm{CDM}$ model is negligible.

\subsection{More general EoS of DM}

Let us consider a more general EoS in the form

$w(z)=B(1+z)^{\alpha}, \quad B<0, \quad \alpha=$ const.

Consider the change in $w$ during the evolution. It is easy to estimate the values of $w\left(z_{r}\right)$ for (11) with different $\alpha$. At $\alpha \geq 0.1$, we get $w\left(z_{r}\right) \approx-0.066 \alpha$. At $\alpha<0$, we have $w\left(z_{r}\right) \approx 0.066 \alpha z_{r}^{\alpha}<0.066 \alpha$. Thus, for $\alpha<3$, the estimate gives $-0.2<w\left(z_{r}\right)<0$. This type of DM significantly distinguishes from DE because of $|w(z)| \ll 1$.
In the modern era, this parameter is equal to $w(0)=B$. For $\alpha>0.2 B \approx-0.066 \alpha$, for $\alpha<-0.2$ $B \approx 0.066 \alpha$. Let us estimate this parameter for the "cosmic dawn". This corresponds to the redshift $z_{g} \approx$ $\approx 11$. At $\alpha \geq 0.2$, we get $w\left(z_{g}\right) \approx-0.066 \alpha 0.01^{\alpha}$, at $\alpha<0$, we have $w\left(z_{r}\right) \approx 0.066 \alpha z_{g}^{\alpha}$. All these values are negative, but close to zero, so the pressure has little effect on the evolution of the Universe and structures in it.

The exception is very low values of $\alpha \ll-1$, which give a large value of $B$ in the modern era. At $\alpha \gg 1$, this problem arises in the recombination era. In the case $\alpha=1$, we have

$\rho_{d m}=\rho_{d m 0}(1+z)^{3} \exp (3 B z)$.

Setting $F\left(z_{r}\right) \approx 0.82$, one can evaluate the combination

$B z_{r} \approx-0.066$.

This is the value of $w$ at the time of the recombination. At the present epoch, we get $w=B \approx$ $\approx-0.066 / 1100 \approx-6 \times 10^{-5}$, and the matter can be considered quite cold. The farthest known galaxy has $z \approx 11$. This corresponds to a value of $w \approx-0.0066$. Thus, the galaxies appeared in the era, when WDM was quite cold in the framework of the considered EoS. However, the structure began to be formed during the period of warmer dark matter.

Note that all obtained estimations for (9) and (11) lie in the parameter interval $-1 / 3<w<0$. I recall that the matter with $w>-1 / 3$ attracts surrounding bodies. But when $w<-1 / 3$, it repels them, by demonstrating the antigravity. This, in particular, is typical of the cosmological constant with $w=-1$ and DE with $w \approx-1$. However, the astronomical observations show that DM attracts both ordinary matter and DM itself.

Naturally, we cannot use $\operatorname{EoS}$ (11) for $\alpha>0$ and huge $z$ significantly exceeding $z_{r}$, since we come to the region with $w(z)<-1$. However, no one considers (11) as a real EoS, but only as an approximation for the period after the recombination epoch. The evolution of the Universe before this epoch lies outside the framework of the considered model.

\section{Decay of DE into Matter}

Is it possible to provide the condition $F\left(z_{r}\right)=0.82$ wi-thout invoking a negative pressure? Suppose that both baryonic and dark matters are pressureless. Nevertheless, the density of matter is now $1: 0.82=1.22$

ISSN 2071-0194. Ukr. J. Phys. 2021. Vol. 66, No. 9 
times more, than it follows from the Eq. (6). In order to explain this, one could assume that its amount has increased from the recombination epoch. Since both types of matter have the same laws of density decreasing in the course of the time, both the decay of DM into ordinary matter and the transition of baryonic matter into dark matter cannot affect the total amount of matter. However, during the transition of DE into matter, an increase in its amount can be obtained without violating the laws of conservation of energy.

So, one could suggest that the contents of the Universe are pressureless, but its density decreased, since the recombination was more slow than that predicted by (6). Moreover, we may assume that there is a source of matter, dark or baryonic, through transition DE into matter. In addition, the process must be sufficiently intense, so that the total amount of matter from the moment of the recombination has increased by approximately $20 \%$.

This possibility is somewhat reminiscent of the now practically forgotten theory, proposed back in 1948 [10, 11]. In it, matter was constantly born "out of nothing", more precisely from the mysterious Cfield (C means creation), maintaining a constant density. Now, we are sure that it is incorrect, and the density of matter is constantly decreasing. However, it can be assumed that different components of the contents of the Universe can transform into one another, keeping its flatness. More specifically, DE can transit into matter.

\section{Conclusions}

The Hubble constant tension can be explained in different ways, from errors in measurements, their processing and interpretation, to the manifestation of some unknown effects. But if we try to explain it, considering the $H_{0}$ values given in [1] to be correct and staying within the framework of cosmological models based on general relativity, in which the Universe contains DE, DM, and cold baryonic matter, then we have few opportunities to choose. Either we should consider DM with negative pressure $-\rho_{d m} c^{2} \ll$ $\ll p_{d m}<0$, which weakly affects the evolution of the Universe and the observed manifestations of DM, or we should admit the mechanism of generation of new matter, for example, by a decay of DE. None of the evolutions of the DE density can explain the differences in the estimates given in [1].
Let me clarify that the mentioning of general relativity may suggest that the conclusions of the article are based on this theory. However, to obtain them, we needed two equations, namely, (1) and (6). Each of them can be obtained within the framework of nonrelativistic cosmology (see [9]). Equation (1) is obtained from classical mechanics and the Newtonian theory of universal gravitation (more precisely, the limit of general relativity for a small curvature of the space-time, generalizing this law), and Eq. (6) follows from the first law of thermodynamics. Therefore, it is impossible to modify the homogeneous isotropic cosmological model by replacing general relativity with another theory, if this does not change the classical mechanics and thermodynamics.

It seems to me that the solution to the problem is more likely associated with the revision and refinement of the estimates of $H_{0}$ for the early and modern Universes, including the consideration of the peculiarities of processing the initial data. But in this article, I focus on the deviations from the $\Lambda$ CDM model and demonstrate that they could theoretically resolve the differences in Hubble constant values obtained from high- and low-redshift observations with a negligible change in the age of the Universe and other parameters. However, they require the introduction of either a new effect, namely, the possibility of the DE transition into matter or the assumption that DM has negative pressure, which excludes the possibility that it consists of currently known particles and even of practically all hypothetical particles considered as candidates for the role of DM.

This work was supported by the National Research Foundation of Ukraine under Project No. 2020.02/0073.

1. L. Verde, T. Treu, A. Riess. Tensions between the early and late universe. Nature Astronomy 3, 891 (2019).

2. Planck Collaboration, N. Aghanim, Y. Akrami et al. Planck 2018 results. VI. Cosmological parameters. A\&A 641 (2020).

3. T.M.C. Abbott, F.B. Abdalla, J. Annis et al. Dark energy survey year 1 results: A precise $\mathrm{H}_{0}$ estimate from DES Y1, $\mathrm{BAO}$, and $\mathrm{D} / \mathrm{H}$ data. MNRAS, 480, 3879 (2018).

4. P. Bode, J.P. Ostriker, N. Turok. Halo formation in warm dark matter models. ApJ 556, 93 (2001).

5. A. Boyarsky, O. Ruchayskiy, D. Iakubovskyi, J. Franse. Unidentified line in X-ray spectra of the Andromeda galaxy and Perseus galaxy cluster. Phys. Rev. Lett. 113, 251301 (2014). 
6. E. Bulbul, M. Markevitch, A. Foster et al. Detection of an unidentified emission line in the stacked X-ray spectrum of galaxy clusters. ApJ 789, 13 (2014).

7. A.D. Dolgov, S.H. Hansen. Massive sterile neutrinos as warm dark matter. Astroparticle Physics 16, 339 (2002)

8. L.D. Landau, E.M. Lifshitz, The Classical Theory of Fields (Butterworth-Heinenann, 1975) [ISBN: 0-7506-2768-9].

9. S. Parnovsky, A. Parnowski. How the Universe Works: Introduction to Modern Cosmology (World Scientific, 2018) [ISBN: 978-981-3234-94-9].

10. H. Bondi, T. Gold. The steady-state theory of the expanding universe. MNRAS 108, 252 (1948).

11. F. Hoyle. A new model for the expanding universe. $M N$ $R A S$ 108, 372 (1948).

Received 09.12.20

\section{С.Л. Парновсъкий}

МОЖЛИВА МОДИФІКАЦІЯ СТАНДАРТНОЇ

КОСМОЛОГІЧНОЇ МОДЕЛІ ДЛЯ РОЗВ'ЯЗАННЯ ПРОБЛЕМИ ЗІ СТАЛОЮ ХАББЛА

Різниця між значеннями сталої Хаббла, отриманими за даними спостережень у ранньому та пізньому Всесвіті, є зна- чною проблемою для сучасної космології. Для розв'язання цієї проблеми розглянуто можливі модифікації плоскої однорідної ізотропної космологічної моделі $\Lambda \mathrm{CDM}$, в якій Всесвіт містить темну енергію, холодну баріонну матерію і темну матерію. Модифіковані моделі базуються на Загальній теорії відносності та задовольняють двом вимогам: (1) значення сталої Хаббла, розраховане із параметра Хаббла за формулами плоскої $\Lambda$ CDM моделі, при рекомбінації має дорівнювати $92 \%$ від значення, розрахованого за спостереженнями з малими червоними зміщеннями; (2) відхилення від моделі $\Lambda \mathrm{CDM}$ не повинно призводити до ефектів, що суперечать астрономічним спостереженням та отриманим 3 них оцінкам. Аналіз показав, що можливостей для вибору небагато. Або ми повинні розглядати темну матерію з від'ємним тиском $-\rho_{d m} c^{2} \ll p_{d m}<0$, котрий слабко впливає на еволюцію Всесвіту та спостережувані прояви темної матерії, або можемо припустити існування якогось механізму генерації нової матерії, наприклад, при розпаді темної енергї.

Ключ о в $i$ слов в: космологія, константа Хаббла, напруга, темна матерія, темна енергія. 